\title{
Bounds on the Capacity of Weakly constrained two-dimensional Codes
}

\section{Forchhammer, Søren}

Published in:

IEEE International Symposium on Information Theory, 2002. Proceedings.

Link to article, DOI:

10.1109/ISIT.2002.1023628

Publication date:

2002

Document Version

Publisher's PDF, also known as Version of record

Link back to DTU Orbit

Citation (APA):

Forchhammer, S. (2002). Bounds on the Capacity of Weakly constrained two-dimensional Codes. In IEEE International Symposium on Information Theory, 2002. Proceedings. IEEE.

https://doi.org/10.1109/ISIT.2002.1023628

\section{General rights}

Copyright and moral rights for the publications made accessible in the public portal are retained by the authors and/or other copyright owners and it is a condition of accessing publications that users recognise and abide by the legal requirements associated with these rights.

- Users may download and print one copy of any publication from the public portal for the purpose of private study or research.

- You may not further distribute the material or use it for any profit-making activity or commercial gain

- You may freely distribute the URL identifying the publication in the public portal

If you believe that this document breaches copyright please contact us providing details, and we will remove access to the work immediately and investigate your claim. 


\title{
Bounds on the Capacity of Weakly Constrained Two-Dimensional Codes
}

\author{
Søren Forchhammer \\ COM, 371 DTU \\ DK-2800 Lyngby, Denmark \\ e-mail: sf@com.dtu.dk
}

\begin{abstract}
Upper and lower bounds are presented for the capacity of weakly constrained twodimensional codes. The maximum entropy is calculated for two simple models of 2-D codes constraining the probability of neighboring $1 \mathrm{~s}$ as an example. For given models of the coded data, upper and lower bounds on the capacity for 2-D channel models based on occurences of neighboring 1s are considered.
\end{abstract}

\section{INTRODUCTION}

Weakly constrained codes in 1-D [1] and constrained codes in 2-D [2] have been considered. We define weakly constrained codes in 2-D, by constraining the values of the probability of subsets of $N$ by $M$ configurations to lie in a given interval. Example 1. A max. probability, $p_{\max }$, is imposed for the occurrence of two neighboring 1's on a 2-D set of binary values.

\section{Bounds on CAPACITIES AND Entropies}

Let $X$ and $Y$ denote the stochastic variables describing the coded data written or sent and the data received, respectively. The achievable rate of the code $X$ over the 2-D channel is given by the mutual entropy

$$
I(X ; Y)=H(X)-H(X \mid Y)=H(Y)-H(Y \mid X)
$$

where $H()$ and $H(\mid)$ denotes the entropy and conditional entropy, respectively. Maximizing $I(X ; Y)$ over the code $X$, given the channel statistics, defines the 2-D channel capacity.

In 1-D, $Y$ is a function of a Markov process for which the entropy $H(Y)$ may be bounded [3]. This approach also yields bounds on $H(X \mid Y)$. In 2-D we consider the class of fields $X$ for which $k$ consecutive rows may be described by a vector Markov process, e.g. Pickard Random Fields (PRF) [4], or a $k$ dimensional vector function of a $k^{\prime}$-dimensional vector Markov process, eg. as in [2].

Given a vector Markov process $X, H(X)$ and $H(Y \mid X)$ may be calculated. The other terms of (1) may be bounded based on applying the 1-D bound [3] to the vector processes. This involves the difference of the entropies on $N$ and $N-1$ rows and generalization of the bound to 2-D. Two examples of bounds are given below based on vector Markov processes. A lower bound based on (1) and bounding $H(X \mid Y)$ using an $m+l+1$ segment $\left(\mathbf{Y}_{t-m}^{t+l}\right)$ of $k$-row vectors, $\mathbf{Y}_{t}$, of $Y$ is given by

$$
I(X, Y) \geq H(X)-H\left(X_{p} \mid \mathbf{Y}_{t-m}^{t+l}\right),
$$

where $m$ and $l$ are positive integers and $X_{p}$ is an element of $X$ whose position coincides with an element of $\mathbf{Y}_{t}$. Now let $t$ be the index of a row by row traversal of $Y$. An upper bound is obtained using (1) and bounding $H(Y)$,

$$
I(X, Y) \leq H\left(Y_{t} \mid F\left(Y^{t-1}\right)\right)-H(Y \mid X),
$$

where $F\left(Y^{t-1}\right)$ is the mapping of the causal part with respect to element $Y_{t}$, e.g. defined by a subset. $H\left(Y_{t} \mid F\left(Y^{t-1}\right)\right)$ may be bounded using the 1-D result [3]. For a given weak constraint and model for $X$, the bounds (2-3) may be optimized over the free parameters of $X$.

For a given constraint defined on the probability of occurence of configurations the capacity of the code, $\max H(X)$ may be bounded by letting a Lagrangian control the probability of a constrained configuration. As for 2-D hard constraints, a band source of width $m$ and extending vertically is introduced defining states having $N-1$ by $m$ elements. Each transition specifies an $N$ by $m$ rectangle by combining the starting and ending states. Let $H(m)$ denote the band entropy given by generating $m$ new elements with each transition. An upper bound (on $\max H(X)$ ) is given by $H(m) / m$ optimized under the given constraint. A lower bound is obtained by concatenating bands of width $m$ now with the additional constraint that the weak constraint is still satisfied after the concatenation of independent bands.

\section{EXPERIMENTS}

In [5], a variation of the PRF was introduced. The probabilities are derived from a 1-D binary Markov chain. For the weak constraint of Ex. 1 and given values of $p_{\max }$, we present the values of entropy $H(X)$ optimized over the parameters of these two models. The MC-based model yielded a slightly higher entropy. Compared with upper and lower bounds calculated using the Lagrangian techniques all the values were close for the same value of $p_{\max }$.

Let the input and output values be binary. As a simple model of the 2-D channel, related to the weak constraint of Ex. 1, we define the error probability to be a function of the neighboring 1's in the input. We present the bounds (2-3) obtained for different functions and different levels of errors, modeling $X$ by the MC-based model [5], as iid binary data and imposing a hard constraint $\left(p_{\max }=0\right)$. The optimized MCbased codes of course yields the highest capacity. The noise level has to be quite significant before the hard constraint code outperforms the iid code.

\section{REFERENCES}

[1] A.J.E.M. Janssen and K.A.S Immink, "Weakly constrained codes," IEEE Trans. Inform. Theory, vol. 46, no. 3, pp. 10341038, May 2000.

[2] S. Forchhammer and J. Justesen, "Entropy bounds for constrained two-dimensional random fields," IEEE Trans. Inform. Theory, vol. 45, no. 1, pp. 118-127, Jan. 1999.

[3] T.M. Cover and J.A. Thomas, Elements of Information Theory, New York: Wiley, 1991.

[4] D.K. Pickard, "A curious binary lattice process," J. Appl. Probab., vol. 14, pp. 717-731, 1977.

[5] J. Justesen, "Fields from Markov chains," submitted to IEEE Trans. Inform. Theory. 